\title{
Retraction Note: Effects of Copper Nanoparticles Located in Different Regions of Polytetrafluoroethylene/Polyimide Blends on the Morphology, Mechanical and Tribological Properties of PTFE Composites
}

\author{
Yuanliang Zhao ${ }^{1,2,3} \cdot$ Xiaowen $^{\mathrm{Q}^{1,2}} \cdot$ Wenli Zhang $^{1,2} \cdot$ Bingli Fan $^{1,2} \cdot$ Qingxiang Yang $^{2,4}$
}

Published online: 30 July 2021

๑) Springer Science+Business Media, LLC, part of Springer Nature 2021

\section{Retraction to: Tribology Letters (2019) 67:18 https://doi.org/10.1007/s11249-018-1128-5}

The Editor-in-Chief has retracted this article. Following publication concerns were raised regarding Fig. 8. Specifically, the figure appears to contain multiple repeated features. The Editor-in-Chief therefore no longer has confidence in the reliability of the data reported in the article. The authors have been offered the chance to submit a revised manuscript for further peer review.

All authors agree to this retraction.

Publisher's Note Springer Nature remains neutral with regard to jurisdictional claims in published maps and institutional affiliations.
The original article can be found online at https://doi.org/10.1007/ s11249-018-1128-5.

Xiaowen Qi

qxw_tougao@163.com

1 School of Mechanical Engineering, Yanshan University, Qinhuangdao 066004, People's Republic of China

2 Aviation Key Laboratory of Science and Technology on Generic Technology of Self-Lubricating Spherical Plain Bearing, Yanshan University, Qinhuangdao 066004, People's Republic of China

3 School of Mechanical Engineering, Shandong University of Technology, Zibo 255049, People's Republic of China

4 School of Material Science and Engineering, Yanshan University, Qinhuangdao 066004, People's Republic of China 